\title{
Involving Support Partners in Obesity Treatment
}

Amy Gorin, The Miriam Hospital/Brown Medical School, Providence, RI, US, Suzanne Phelan, The Miriam Hospital/Brown Medical School, Providence, RI, US Deborah Tate, The Miriam Hospital/Brown Medical School, Providence, RI, US

Nancy Sherwood, Department of Epidemiology, University of Minnesota, Minneapolis, MN, US Robert Jeffery, Department of Epidemiology, University of Minnesota, Minneapolis, MN, US Rena Wing, The Miriam Hospital/Brown Medical School, Providence, RI, US

Acknowledgement: This research was supported by National Heart, Lung, and Blood Institute Grants HL41330 and HL41322.

\begin{abstract}
In this study, the authors examined whether the number or success of weight loss partners influences participants' outcomes in behavioral weight loss treatment. Overweight participants $(n=109)$ assigned to an exercise intensive group in a larger trial were encouraged to invite up to 3 partners to attend treatment. Weight losses at 6,12, and 18 months were not associated with the number of partners (0-3) but were associated with the weight loss success of the partners. Participants with at least 1 successful partner (weight loss $\geq 10 \%$ at 6 months) lost significantly ( $\mathrm{p}=.004$ ) more weight at 6,12 , and 18 months than those with no successful partners and those without partners. Interclass correlations of weight change between participants and their partner(s) were strong at all time points $(\mathrm{ps}<.01)$. Support partners appear to only be beneficial in obesity treatment when partners themselves lose weight.
\end{abstract}


Involving support partners in behavioral weight control treatment is one of the few strategies shown to improve weight loss maintenance (Black, Gleser, \& Kooyers, 1990; Jeffery et al., 2000). For example, in a prior study, Wing and Jeffery (1999) reported that overweight individuals who brought friends with them to a behavioral weight control program were more likely to maintain their weight loss over 6 months of follow-up than individuals who participated in treatment alone. Although bringing support partners to treatment represents a promising way to improve both initial weight loss and maintenance of weight loss, there have been exceptions in the literature (e.g., Brownell \& Stunkard, 1981; Wing, Marcus, Epstein, \& Jawad, 1991). A meta-analysis of 13 studies examining the impact of spouse involvement in weight loss treatment reported only a modest, short-term effect of spouse involvement (Black et al., 1990). It remains unclear in the social support literature how many support partners it takes to optimize weight loss outcomes and whether partners need to lose weight themselves to be an effective form of support. In the present study, we examined these questions in overweight individuals who participated in a larger randomized controlled trial.

\section{Method}

\section{Participants}

A total of 202 participants were recruited through newspaper advertisements that sought overweight individuals interested in weight loss. Participants had to be 25-50 years old and 14$32 \mathrm{~kg}$ overweight according to actuarial norms (Metropolitan Life Insurance, 1983). Participants also had to report no serious concurrent medical or psychological problems that might interfere with treatment. Eligible participants were randomly assigned to receive a standard behavioral treatment with either a $1,000 \mathrm{kcal}$ per week or 2,500 kcal per week exercise goal. In both conditions, treatment meetings were held weekly for the first 6 months, biweekly from 6 to 12 months, and monthly from 12 to 18 months. In addition, a 1,000-1,200 kcal, 20\% fat diet was prescribed (see Jeffery, Wing, Sherwood, \& Tate's, 2003, study for more detailed intervention descriptions). In the present investigation, we focused exclusively on participants in the high physical activity group $(n=109)$ who were encouraged, but not required, to invite up to three support partners to join the study. The rationale for including partners in this treatment group was to provide additional support to participants to help them achieve the higher exercise goals. Support partners had to be between the ages of 21-50 years and 7-32 kg overweight (which represented a slightly lower body weight than participants). Support partners and participants were treated identically in terms of screening, assessment, and intervention.

\section{Assessments}

The following information was collected from participants and support partners at baseline, 6 , 12 , and 18 months, unless otherwise noted.

\section{Demographic information}

Basic demographic information (i.e., age, gender, ethnicity, education, marital status) was collected at baseline. 


\section{Weight and height}

Weight was measured on a calibrated scale. Baseline height was measured with a wall-mounted ruler at baseline and used to calculate body mass index $\left(\mathrm{BMI} ; \mathrm{kg} / \mathrm{m}^{2}\right)$.

\section{Diet and physical activity}

The Block Food Frequency Questionnaire (Block, Woods, Potosky, \& Clifford, 1990), which asks participants to indicate the frequency of consumption and typical portion size of 97 foods, was used to estimate current total daily energy intake. Although underreporting is a problem with all modes of self-reported intake, the Block Food Frequency Questionnaire has been found to closely correspond with dietary records, and it has been validated against 3-day records (Block et al., 1986, 1990). The Paffenbarger Activity Questionnaire (Paffenbarger, Wing, \& Hyde, 1978), which asks about the number of stairs climbed, blocks walked, and sports activities, was used to provide an estimate of calories expended per week in overall leisure time activity. Previous studies have reported that changes in exercise on the Paffenbarger Activity Questionnaire are predictive of weight change (Harris, French, Jeffery, McGovern, \& Wing, 1994).

\section{Statistics}

Independent $t$ tests (for continuous measures) and chi-square analyses (for categorical variables) were used to compare participants and support partners, as well as completers and noncompleters, on demographic variables. A repeated measures analysis of variance in which the baseline value was carried forward in the case of missing data was used to compare individuals with successful support partners, unsuccessful support partners, and no partners on weight loss over time (Ware, 2003). Post hoc tests were performed with Bonferroni correction. Interclass correlations (ICCs) were used to examine the relationship between participants' and support partners' weight and behavior changes.

\section{Results}

Of the 109 participants, $94 \%$ completed the 6-month assessment, compared with $79 \%$ at 12 months and $80 \%$ at 18 months. Participants who completed the 18 -month study were significantly older than noncompleters $(44.0 \pm 5.7$ vs. $40.7 \pm 6.7$ years $), t(107)=2.4, p=.02$. No significant differences in initial BMI, gender, marital status, ethnicity, employment, or number of support partners were observed.

Nearly half of the participants (49\%) brought support partners. Participants who brought partners did not differ from participants who did not bring partners on demographic variables (i.e., gender, education, marital status, ethnicity, age, BMI). The majority of participants with partners brought one partner (71.4\%); 19.6\% brought two partners; and 8.9\% brought three partners. Participants and partners were similar demographically, on baseline eating and exercise behaviors, and in the amount of change in weight, eating, and exercise over time (see Table 1). Partners were, however, more likely than participants to be women $(80.5 \%$ vs. $55.0 \%, p \leq .01)$. Overall, most partners were described as friends, neighbors, or coworkers $(65.5 \%)$ by participants; $17 \%$ were the participant's spouse; and $13 \%$ were other family members of the participant.

There were no significant differences in weight losses at 6,12 , and 18 months between participants who brought partners $(8.9 \pm 7.2,6.5 \pm 7.6,6.5 \pm 7.4 \mathrm{~kg})$ and participants who did not bring partners $(7.8 \pm 7.1$, 
$5.9 \pm 7.5,4.6 \pm 7.9 \mathrm{~kg}$ ). The number of support partners brought to treatment (i.e., $0-3)$ was also not significantly related to participants' weight loss at $6(\mathrm{r}=.07, \mathrm{p}=.35), 12(\mathrm{r}=.09, \mathrm{p}=.25)$, or $18(\mathrm{r}=.09, \mathrm{p}$ $=.29$ ) months. However, the weight loss success of the support partner(s) was associated with participants' treatment outcomes. Because weight losses of $>10 \%$ of initial body weight have been shown to produce clinically significant improvements in cardiovascular risk factors (National Heart, Lung, and Blood Institute, 1998; Wadden \& Foster, 2000), we defined successful partners as partners who at the 6-month assessment had lost at least $10 \%$ of their initial body weight. Using this definition, we found that participants who had at least one successful partner $(\mathrm{n}=30)$ lost significantly more weight, $\mathrm{F}(6,318)=$ $3.2, \mathrm{p}=.004(\eta 2=.06)$, at 6,12 , and 18 months $(11.4 \pm 6.3,10.4 \pm 7.2,8.6 \pm 7.1 \mathrm{~kg})$ than participants with no successful partners $(\mathrm{n}=26 ; 6.2 \pm 7.1,5.2 \pm 7.6,4.1 \pm 7.0 \mathrm{~kg})$ and those who did not identify a partner $(\mathrm{n}=53 ; 7.8 \pm 7.3,5.4 \pm 7.7,4.2 \pm 8.1 \mathrm{~kg})$. Participants without partners and those with partners who were unsuccessful did not differ significantly on weight change at any of the time points. Similar results were found when we examined percentage changes in initial body weight (see Figure 1). Having more than one successful partner did not further improve participants' weight loss at any time point.

The ICCs of weight change between participants and their support partner(s) were strong at 6,12 , and 18 months (ICC $=.51, p=.003 ; .57, p=.001$; and $.46, p=.01$; respectively), underscoring the collinearity in weight change between participants and their support partners. A significant ICC was also observed between participants and their support partner(s) on changes in caloric intake at 6 months (ICC $=.52, p$ $=.03)$ but not at $12(\mathrm{ICC}=.11, p=.39)$ or $18(\mathrm{ICC}=.16, p=.30)$ months. A trend was observed on changes in exercise at 18 months (ICC $=.40, p=.06)$, but no effects were found at 6 (ICC $=.08, p=.60)$ or 12 (ICC $=.23, p=.73$ ) months. Significant ICCs were also observed between participants and their support partner(s) on total number of food diaries completed at $6(\mathrm{ICC}=.68, p=.0001), 12(\mathrm{ICC}=.67, p=.0001)$, and $18(\mathrm{ICC}=.68, p=.0001)$ months. No significant relationship was found between participants' and their partner(s)' dropout status at 6,12 , or 18 months.

There were no demographic or behavioral differences at baseline between support partners who went on to be successful at weight loss versus those who did not (variables examined included age, gender, education, marital status, relationship to participant [family or friend], baseline BMI, exercise levels, and caloric and fat intake). Thus, consistent with the general treatment literature, baseline variables did not allow us to predict which partners would subsequently be successful.

\section{Discussion}

Prior research has found that bringing a friend and/or family member to behavioral weight loss treatment improves short- and long-term weight loss outcomes (Black et al., 1990; Wing \& Jeffery, 1999), but other research has found no effect of peer support (e.g., Brownell \& Stunkard, 1981; Wing et al., 1991). In the present study, we may explain these mixed findings by suggesting that involving support partner(s) in obesity treatment is likely to result in better weight losses only when the support partners are themselves successful at losing weight. The overall number of partners $(0-3)$ was not associated with participants' weight losses at 6,12 , and 18 months. Rather, the best weight losses were observed in participants who brought at least one support partner who went on to lose at least $10 \%$ of his or her initial body weight in the first 6 months of treatment. Although the directionality of this relationship cannot be determined, the important finding is that the weight losses of the participant and partner(s) clustered together, indicating that participants whose partners are successful at weight loss are more likely to be successful themselves.

The weight loss behaviors of participants and partners also clustered together. Of particular note, a strong relationship was observed between participants and partners in terms of adherence to treatment, as measured by the number of food diaries completed. Prior studies, such as Wing et al.'s (1991) examination of a family-based approach to weight loss, have shown similar correlations in dietary changes between marital partners participating in treatment together. 
The present study was not a randomized controlled trial, and participants were limited to those enrolled in an exercise-intensive program. Controlled research designs are needed to determine directionality of participant-partner success and to better identify the characteristics that differentiate successful from unsuccessful partners. The strengths of the study include the long-term follow-up and the evaluation of participants with differing numbers of support partners.

Extending the focus of weight loss treatment from the individual participant to the individual participant plus his or her support partner(s) is an attractive treatment option because of the positive effect it has on weight loss maintenance as demonstrated by Wing and Jeffery (1999). In this study, we suggest that participants, in terms of weight loss, are only as good as the company they keep; thus, they should be encouraged to invite support partner(s) who are motivated to lose weight themselves. 


\section{Table 1: Baseline Characteristics and Changes in Weight and Weight-Related Behaviors in Participants and Partners}

\begin{tabular}{|c|c|c|}
\hline Characteristic & $\begin{array}{c}\text { Participants } \\
(n=109)\end{array}$ & $\begin{array}{c}\text { Parnters } \\
(n=77)\end{array}$ \\
\hline Age & $41.5 \pm 5.2$ & $40.5 \pm 7.2$ \\
\hline \multicolumn{3}{|l|}{ Gender } \\
\hline Women & 55.0 & $80.5^{*}$ \\
\hline Men & 45.0 & $19.5^{*}$ \\
\hline \multicolumn{3}{|l|}{ Ethnicity (\%) } \\
\hline Caucasian & 82.1 & 79.2 \\
\hline African American & 11.8 & 12.0 \\
\hline Hispanic & 3.6 & 3.3 \\
\hline Native American & 2.5 & 5.5 \\
\hline $\mathrm{BMI}$ & $31.1 \pm 2.7$ & $30.4 \pm 3.8$ \\
\hline Caloric intake (kcal/day) & $2,189.0 \pm 1,066.0$ & $2,124.0 \pm 1,045.0$ \\
\hline \multicolumn{3}{|l|}{ Change in caloric intake } \\
\hline \multicolumn{3}{|l|}{ (kcal/day) } \\
\hline $0-6$ months & $-573.4 \pm 762.9$ & $-609.8 \pm 717.8$ \\
\hline $0-12$ months & $-543.1 \pm 959.1$ & $-482.6 \pm 822.5$ \\
\hline $0-18$ months & $-486.4 \pm 1,027.3$ & $-561.2 \pm 772.8$ \\
\hline Exercise level (kcal/week) & $1,278.0 \pm 1,369.0$ & $1,283.0 \pm 1,530.0$ \\
\hline \multicolumn{3}{|l|}{ Change in exercise level } \\
\hline \multicolumn{3}{|l|}{ (kcal/week) } \\
\hline $0-6$ months & $1,138.3 \pm 1,893.2$ & $1,078.6 \pm 1,767.4$ \\
\hline $0-12$ months & $659.9 \pm 1,547.5$ & $1,030.5 \pm 1,685.8$ \\
\hline $0-18$ months & $1,170.3 \pm 1,974.9$ & $969.6 \pm 2,200.5$ \\
\hline \multicolumn{3}{|l|}{ Weight Change (kg) } \\
\hline $0-6$ months & $-8.4 \pm 7.2$ & $-7.7 \pm 7.0$ \\
\hline $0-12$ months & $-6.7 \pm 7.8$ & $-6.1 \pm 7.4$ \\
\hline $0-18$ months & $-5.4 \pm 7.6$ & $-4.9 \pm 7.9$ \\
\hline
\end{tabular}

Note. Unless otherwise indicated, values indicate means plus/minus standard deviations. BMI = body mass index.

$* p \leq .01$. 


\section{Figure 1}

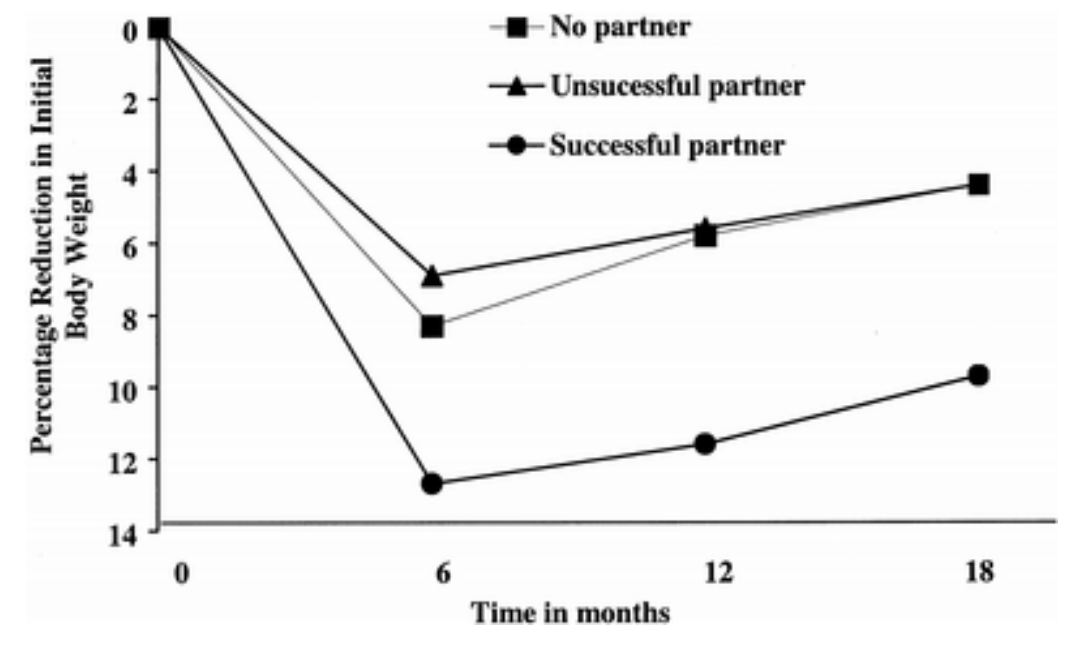

Percentage of weight loss over time among participants without partners, with successful partners, and with unsuccessful partners. Successful was defined as having lost $\geq 10 \%$ of initial body weight at 6 months. 


\section{References}

1. Black, D. R., Gleser, L. J., \& Kooyers, K. J. (1990). A meta-analytic evaluation of couples weight-loss programs. Health Psychology, 9, 330-347.

2. Block, G., Hartman, A. M., Dresser, C. M., Carroll, M. D., Gannon, J., \& Gardner, L. (1986). A data-based approach to diet questionnaire design and testing. American Journal of Epidemiology, 124, 453-469.

3. Block, G., Woods, M., Potosky, A., \& Clifford, C. (1990). Validation of a selfadministered diet history questionnaire design using multiple diet records. Journal of Clinical Epidemiology, 43, 1327-1335.

4. Brownell, K. D., \& Stunkard, A. J. (1981). Couples training, pharmacotherapy, and behavior therapy in the treatment of obesity. Archives of General Psychiatry, 38, 12241229.

5. Harris, J. K., French, S. A., Jeffery, R. W., McGovern, P. G., \& Wing, R. R. (1994). Dietary and physical activity correlates of long-term weight loss. Obesity Research, 2 , 307-313.

6. Jeffery, R. W., Drewnowski, A., Epstein, L. H., Stunkard, A. J., Wilson, G. T., Wing, R. R., \& Hill, J. (2000). Long-term maintenance of weight loss: Current status. Health Psychology, 19, 5-16.

7. Jeffery, R. W., Wing, R. R., Sherwood, N. E., \& Tate, D. F. (2003). Physical activity and weight loss: Does prescribing higher physical activity goals improve outcome?American Journal of Clinical Nutrition, 78, 684-689.

8. Metropolitan Life Insurance. (1983). Metropolitan height and weight tables. Statistical Bulletin of Metropolitan Life Insurance Company, 64, 2-9.

9. National Heart, Lung, and Blood Institute. (1998). Clinical guidelines on the identification, evaluation, and treatment of overweight and obesity in adults: The evidence report. Obesity Research, 6, 51S-210S.

10. Paffenbarger, R. S., Wing, A. L., \& Hyde, R. T. (1978). Physical activity as an index of heart attack risk in college alumni. American Journal of Epidemiology, 108, 161-175.

11. Wadden, T. A., \& Foster, G. D. (2000). Behavioral treatment of obesity. Medical Clinics of North America, 84, 441-462.

12. Ware, J. H. (2003). Interpreting incomplete data in studies of diet and weight loss. New England Journal of Medicine, 348, 2136-2137.

13. Wing, R. R., \& Jeffery, R. W. (1999). Benefits of recruiting participants with friends and increasing social support for weight loss and maintenance. Journal of Consulting and Clinical Psychology, 67, 132-138.

14. Wing, R. R., Marcus, M. D., Epstein, L. H., \& Jawad, A. (1991). A family-based approach to the treatment of obese Type 2 diabetic patients. Journal of Consulting and Clinical Psychology, 59, 156-162. 